Meta

Journal des traducteurs

Translators' Journal

\title{
Vers un produit unifié en terminologie et en documentation : le thésaurus terminologique
}

\section{Louise Larivière}

Volume 34, numéro 3, septembre 1989

1. Actes du Colloque Les terminologies spécialisées: Approches quantitative et logico-sémantique et 2 . Actes du Colloque Terminologie et Industries de la langue

URI : https://id.erudit.org/iderudit/003400ar

DOI : https://doi.org/10.7202/003400ar

Aller au sommaire du numéro

Éditeur(s)

Les Presses de l'Université de Montréal

ISSN

0026-0452 (imprimé)

1492-1421 (numérique)

Découvrir la revue

Citer cet article

Larivière, L. (1989). Vers un produit unifié en terminologie et en documentation : le thésaurus terminologique. Meta, 34(3), 457-467. https://doi.org/10.7202/003400ar
Résumé de l'article

Les terminologies et les thésaurus documentaires présentent suffisamment d'affinités au plan de leur contenu et de leur structure pour qu'on puisse envisager la création d'un seul répertoire unifié. Un tel produit pourrait prendre la forme d'un thésaurus terminologique qui servirait tant à l'indexation et au repérage des documents qu'à la consultation des données terminologiques et dont la confection s'effectuerait à partir des méthodes utilisées en terminologie et en documentation. Pour ce faire, les descripteurs des thésaurus devront acquérir la spécificité du terme et s'accompagner d'une définition descripteurs des thésaurus devront acquérir la spécificité du terme et s'accompagner d'une définition tandis que les terminologies devront adopter la structure des thésaurus : système de relations et plan
classement. En s'appuyant sur ces principes, on présente un modèle de thésaurus terminologique classement. En s'appuyant sur ces principes,

D'entrée de jeu, Sparck Jones et Kay (1973: 1) affirment, dans l'introduction à leur étude sur les rapports entre la linguistique et les sciences de l'information, que ces deux disciplines ont suffisamment d'affinités pour faire bon ménage ("are bedfellows"). La linguistique, en effet, a pour objet de décrire les langues naturelles qui ont pour fonction, entre autres, de transmettre de l'information, alors que les sciences de l'information ont pour objet de repérer de l'information traduite dans des documents écrits en langue naturelle. Tout en reconnaissant l'existence de recoupements entre les deux disciplines, ces auteurs s'étonnent, néanmoins, de leur manque d'interpénétration et s'interrogent sur les causes d'un tel fait et sur les mesures à prendre pour y remédier. Les recoupements sont si évidents, selon eux, qu'ils se sur les mesures à prendre pour y remédier. Les recoupements sont si évidents, selon eux, qu'ils se
demandent, même, si les sciences de l'information ne devraient pas constituer une sous-discipline de la demandent, même, si les sciences de l'information ne devraient pas constituer une sous-discipline de la
linguistique ou la linguistique faire partie des sciences de l'information. Mais tel n'est pas leur but de répondre à cette question. Ils cherchent plutôt à savoir ce que ces deux disciplines peuvent s'apporter mutuellement et quel degré d'interpénétration elles ont atteint ou peuvent atteindre.

S'est-on, par ailleurs, posé les mêmes questions au sujet de la terminologie et de la documentation? Depuis une quinzaine d'années, un certain rapprochement s'est amorcé entre terminologues et spécialistes en information documentaire. Dans les publications canadiennes des années 70 (articles de revues et actes de colloque), on s'est surtout arrêté sur la façon dont la fonction recherche de la documentation pouvait optimiser le rendement des activités terminologiques et traductionnelles de plus en plus intenses et complexes1, mais on a démontré peu d'intérêt pour la documentation dans sa fonction analyse. On croit même, dans certains milieux, que la terminologie et la documentation constituent "deux univers séparés dont les concepts de base sont bien distincts, la documentation s'occupant de classes et la terminologie de notions à structurer et à dénommer"2. Quelques auteurs se sont attardés, cependant, sur un aspect de la fonction analyse de la documentation, soit la classification, et ont préconisé le répertoire de type thésaurus comme système de classement des données terminologiques à l'intérieur d'une banque de données3.

C'est donc vers l'Europe surtout et les États-Unis qu'il faut se tourner pour trouver des écrits dans lesquels on compare les démarches utilisées en terminologie et en documentation pour produire les répertoires terminologiques, d'une part, et les répertoires documentaires que sont les thésaurus, d'autre part, pour en conclure qu'elles possèdent suffisamment d'affinités pour tendre progressivement vers un produit unifié4.

Un tel produit unifié pourrait prendre la forme d'un thésaurus terminologique qui servirait tant à l'indexation et au repérage des documents qu'à la consultation de données terminologiques et dont la confection s'effectuerait à partir des méthodes utilisées en terminologie et en documentation.

C'est ce à quoi vise cette communication : 1) justifier la création d'un tel répertoire en comparant les produits fabriqués traditionnellement, l'un par les terminologues (les terminologies) et l'autre par les documentalistes (les thésaurus), du point de vue de leur finalité, de leur contenu, de leur structure pou en faire ressortir les lacunes et les insuffisances; 2) décrire son fonctionnement à partir d'un modèle appliqué aux documents professionnels.
Ce document est protégé par la loi sur le droit d'auteur. L’utilisation des services d’Érudit (y compris la reproduction) est assujettie à sa politique d'utilisation que vous pouvez consulter en ligne.

https://apropos.erudit.org/fr/usagers/politique-dutilisation/ 


\section{VERS UN PRODUIT UNIFIÉ EN TERMINOLOGIE ET EN DOCUMENTATION LE THÉSAURUS TERMINOLOGIQUE}

LOUISE LARIVIERE

Collège universitaire de Saint Boniface, Winnipeg, Canada

RÉSUMÉ

Les terminologies et les thésaurus documentaires présentent suffisamment d'affinités au plan de leur contenu et de leur structure pour qu'on puisse envisager la création đ'un seul répertoire unifié. Un tel produit pourrait prendre la forme d'un thésaurus terminologique qui servirait tant à l'indexation et au repérage des documents qu'à la consultation des données terminologiques et dont la confection s'effectuerait à partir des méthodes utilisées en terminologie et en documentation. Pour ce faire, les descripteurs des thésaurus devront acquérir la spécificité du terme et s'accompagner d'une définition tandis que les terminologies devront adopter la structure des thésaurus: système de relations et plan de classement. En s'appuyant sur ces principes, on présente un modèle de thésaurus terminologique appliqué aux documents professionnels.

D'entrée de jeu, Sparck Jones et Kay (1973: 1) affirment, dans l'introduction à leur étude sur les rapports entre la linguistique et les sciences de l'information, que ces deux disciplines ont suffisamment d'affinités pour faire bon ménage («are bedfellows»). La linguistique, en effet, a pour objet de décrire les langues naturelles qui ont pour fonction, entre autres, de transmettre de l'information, alors que les sciences de l'information ont pour objet de repérer de l'information traduite dans des documents écrits en langue naturelle. Tout en reconnaissant l'existence de recoupements entre les deux disciplines, ces auteurs s'étonnent, néanmoins, de leur manque d'interpénétration et s'interrogent sur les causes d'un tel fait et sur les mesures à prendre pour y remédier. Les recoupements sont si évidents, selon eux, qu'ils se demandent, même, si les sciences de l'information ne devraient pas constituer une sous-discipline de la linguistique ou la linguistique faire partie des sciences de l'information. Mais tel n'est pas leur but de répondre à cette question. Ils cherchent plutôt à savoir ce que ces deux disciplines peuvent s'apporter mutuellement et quel degré d'interpénétration elles ont atteint ou peuvent atteindre.

S'est-on, par ailleurs, posé les mêmes questions au sujet de la terminologie et de la documentation? Depuis une quinzaine d'années, un certain rapprochement s'est amorcé entre terminologues et spécialistes en information documentaire. Dans les publications canadiennes des années 70 (articles de revues et actes de colloque), on s'est surtout arrêté sur la façon dont la fonction recherche de la documentation pouvait optimiser le rendement des activités terminologiques et traductionnelles de plus en plus intenses et complexes', mais on a démontré peu d'intérêt pour la documentation dans sa fonction analyse. On croit même, dans certains milieux, que la terminologie et la documentation constituent «deux univers séparés dont les concepts de base sont bien distincts, la documentation s'occupant de classes et la terminologie de notions à structurer et à dénommer» ${ }^{2}$. Quelques auteurs se sont attardés, cependant, sur un aspect de la fonction analyse de la documentation, soit la classification, et ont préconisé le répertoire de type 
thésaurus comme système de classement des données terminologiques à l'intérieur d'une banque de données ${ }^{3}$.

C'est donc vers l'Europe surtout et les États-Unis qu'il faut se tourner pour trouver des écrits dans lesquels on compare les démarches utilisées en terminologie et en documentation pour produire les répertoires terminologiques, d'une part, et les répertoires documentaires que sont les thésaurus, d'autre part, pour en conclure qu'elles possèdent suffisamment d'affinités pour tendre progressivement vers un produit unifié4.

Un tel produit unifié pourrait prendre la forme d'un thésaurus terminologique qui servirait tant à l'indexation et au repérage des documents qu'à la consultation de données terminologiques et dont la confection s'effectuerait à partir des méthodes utilisées en terminologie et en documentation.

C'est ce à quoi vise cette communication : 1) justifier la création d'un tel répertoire en comparant les produits fabriqués traditionnellement, l'un par les terminologues (les terminologies) et l'autre par les documentalistes (les thésaurus), du point de vue de leur finalité, de leur contenu, de leur structure pour en faire ressortir les lacunes et les insuffisances; 2) décrire son fonctionnement à partir d'un modèle appliqué aux documents professionnels.

\section{A - COMPARAISON ENTRE TERMINOLOGIES ET THÉSAURUS}

On peut définir terminologies et thésaurus comme suit.

Les terminologies sont des répertoires ou des listes de termes spécialisés d'un domaine particulier, accompagnés d'une définition et pouvant être regroupés selon un classement alphabétique ou un classement thématique. Fabriqués par des terminologues, de concert avec des spécialistes d'un domaine, ils sont destinés à la consultation par des chercheurs, des langagiers (traducteurs, rédacteurs, terminologues), des professeurs enseignant une discipline donnée ou par tout autre usager qui s'interroge sur la pertinence d'un terme à des fins de communication. Ils sont donc des intermédiaires entre les référents (ou la réalité des objets) et les utilisateurs. Ils peuvent également servir aux documentalistes dans l'établissement des thésaurus pour préciser le sens d'un descripteur.

Quant aux thésaurus, ils sont des répertoires ou listes d'autorité constitués d'unités (descripteurs et non-descripteurs) appartenant à un domaine particulier et de relations (hiérarchiques, préférentielles et associatives) qui précisent leur environnement sémantique, et regroupés selon divers modes de présentation. Fabriqués par des documentalistes, de concert avec des spécialistes d'un domaine, ils servent à caractériser le contenu d'un document (termes d'indexation) et le contenu des questions posées par les utilisateurs lors d'une recherche documentaire (termes de la question) en vue d'un repérage rapide et efficace des documents souhaités. Ils sont donc des intermédiaires entre les documents et les utilisateurs. Utilisés, avant tout, par des documentalistes lors des activités d'indexation et de repérage, ils peuvent également être consultés par les chercheurs et les langagiers, en particulier par les terminologues, comme documents de référence lors d'un travail terminologique «pour mettre au point la structuration du domaine et pour l'établissement des listes de catégories notionnelles» (Auger, 1978: 20).

Il ressort de ces définitions que les terminologies et les thésaurus diffèrent quant à leur finalité, leur contenu et leur structure.

\section{QUANT À LEUR FINALITÉ}

Comme les deux répertoires poursuivent des buts différents, les terminologies servant à inventorier les termes d'un domaine et à décrire les notions désignées par ces termes au moyen de définitions (Boutin-Quesnel, 1985:29) et les thésaurus servant à 
caractériser le contenu des documents et des questions à l'aide de descripteurs et de relations, il va se soi que leur contenu (respectivement termes et descripteurs) et leur structure (respectivement définitions et relations) seront différents.

Il n'en demeure pas moins, toutefois, que les terminologies et les thésaurus sont tous deux des répertoires ou des listes constitués d'unités lexicales appartenant à un domaine particulier du savoir. Par conséquent, il arrive, comme c'est souvent le cas, que l'on crée l'un et l'autre répertoire pour un même domaine donné. N'y aurait-il pas lieu, alors, pour éviter le double emploi, d'envisager la création d'un seul répertoire qui satisfasse, à la fois, à la finalité des thésaurus et des terminologies même si, pour ce faire, l'on doit modifier quelque peu le contenu et la structure des uns et des autres?

Pour répondre à cette question, il est nécessaire, d'abord de voir si ce contenu et cette structure sont réconciliables.

\section{QUANT À LEUR CONTENU}

Les unités contenues dans les terminologies consistent en des termes, alors que celles contenues dans les thésaurus consistent en des descripteurs. Termes et descripteurs diffèrent par les points suivants : 1) les termes appartiennent à la langue spécialisée alors que les descripteurs peuvent appartenir soit à la langue spécialisée, soit à la langue commune; 2) les termes peuvent être extraits des documents, donc constituer des unités existantes, ou résulter d'une création néologique; quant aux descripteurs, ils sont généralement des unités connues des utilisateurs puisqu'ils sont établis à partir de documents d'analyse (ouvrages du domaine ou questions des utilisateurs) et des documents de référence. En aucun cas, ils ne sont créés; 3) termes et descripteurs n'ont pas les mêmes référents : par exemple, le terme RAPPORT, dans une terminologie, renverrait à un certain objet en tant qu'élément de la réalité, tandis que le descripteur RAPPORT, dans un thésaurus, choisi parmi d'autres unités équivalentes comme indice ou formule d'indexation, renverrait à des documents qui traitent de cet élément de la réalité5; 4) le terme est une unité monoréférentielle i.e. qu'il ne renvoie qu'à une seule réalité ou référent tandis que le descripteur est une unité préférentielle choisie parmi d'autres pour représenter plus d'une réalité (bien qu'il arrive parfois que le descripteur ne renvoie qu'à une seule réalité). C'est pourquoi le descripteur n'a pas la précision du terme.

Malgré ces différences, les termes et les descripteurs partagent certaines caractéristiques: 1) ils sont tous deux des unités signifiantes monosémiques, simples ou complexes, qui représentent, sans ambiguïté ou de façon univoque, une notion/concept à l'intérieur d'un même domaine (AFNOR, 1981b: 3 et 18; Boutin-Quesnel et al., 1985: $20)$; 2) ils sont tous deux, également, «des signes du langage naturel et non pas des codages artificiels (numériques ou alphanumériques)» (Maniez, 1977 : IV-44); 3) ils suivent, finalement, de près «les habitudes langagières de la spécialité traitée» (Maniez, 1977: IV-44).

Malgré leurs différences, les termes et les descripteurs ont, toutefois, suffisamment d'affinités pour tenter de les rapprocher, d'autant plus que, de toutes les unités lexicales utilisées dans les divers langages documentaires, seuls les descripteurs des thésaurus partagent avec les termes les trois caractéristiques déjà mentionnées. De plus, le vocabulaire spécialisé devenant de plus en plus spécifique, les descripteurs des thésaurus ne peuvent se permettre d'être trop vastes (v. l'exemple signalé à la note 6), sinon on ne peut juger du contenu réel d'un document et on obtient trop de «silence» lors du repérage des documents dû à un manque de spécificité au moment de l'indexation.

Aussi, les descripteurs se doivent de gagner en précision et de s'apparenter de plus en plus aux termes, même si un «excès de profondeur ou de finesse» pourrait, à l'occa- 
sion, nuire à la recherche. Rien n'empêche, toutefois, de limiter cette dernière à un certain niveau de spécificité i.e. en ne descendant pas trop bas à l'intérieur d'une hiérarchie même si plusieurs niveaux inférieurs sont répertoriés dans les listes des thésaurus.

Il n'en demeure pas moins que les termes complexes à plusieurs éléments, du genre «service de transmission de données à commutation de circuit» (Clas, 1986: 382-383) seraient trop spécifiques pour une recherche documentaire. En effet, l'établissement de terminologies bilingues, à partir de l'anglais comme langue de départ, a entraîné la création de néologismes en français qui ne se retrouvent pas dans la documentation française, ce qui ne facilite pas une recherche qui s'appuie sur des unités d'indexation extraites des documents. Cependant, comme le mentionnent les auteurs cités plus haut, ces «composés périphrastiques présentent un modèle transitoire» et posent «des problèmes de découpage en constituants pour la mini-banque (i.e. une banque de terminologie sur micro-ordinateur)» et se doivent d'être découpés en unités plus petites: ex. «service de transmis sion», «transmission de données», «transmission à commutation de circuit», «commutation de circuit», unités qui pourraient être post-coordonnées, au besoin, lors d'une recherche documentaire.

\section{QUANT À LEUR STRUCTURE}

Dans les terminologies, les termes sont accompagnés d'une définition alors que, dans les thésaurus, les descripteurs sont accompagnés d'unités avec lesquelles ils entretiennent diverses relations (d'équivalence, hiérarchiques et associatives).

Les définitions et les relations jouent, toutefois, un rôle semblable bien qu'il s'exprime différemment. La définition qui consiste en un rapport entre un défini (definiendum) et un définissant (definiens) fait ressortir les caractéristiques du défini, en l'opposant aux autres définis qui s'en rapprochent, par la mise en valeur de ses traits sémantiques pertinents (Dahlberg, 1981: 17 et 1983:20-21). Le premier énoncé du définissant (genre prochain ou tout) déterminé à partir d'un classement thématique hiérarchisé corrrespond, dans les relations, à l'unité de la hiérarchie supérieure (terme générique ou tout), alors que le reste de la définition, que représentent les caractéristiques spécifiques du défini, correspond, dans les relations, aux unités inférieures (termes spécifiques ou parties) et aux termes associés (Dovbenko et Umanski, 1980: 10). Les relations ne sont pas toutes, cependant, nécessairement établies de cette façon; de plus, bien qu'elles permettent, à l'aide de rapprochements, d'identifier le sens d'une unité, elles n'arrivent jamais à en préciser le sens aussi clairement que le ferait une définition.

Par ailleurs, bien que des définitions n'apparaissent pas comme telles dans les thésaurus, on y utilise, à l'occasion, des notes d'application ou d'explication pour préciser/définir les modalités d'emploi d'un descripteur afin de ne laisser subsister aucune ambiguïté du langage naturel qui pourrait gêner au moment de l'indexation ou de la rédaction d'une question. Elles se limitent à certains descripteurs pour éviter qu'ils ne soient interprétés différemment selon les domaines ou lorsqu'un terme a un sens particulier dans certains ouvrages de référence (AFNOR, 1981b: 6-7).

Ces notes sont toutefois insuffisantes. Le vocabulaire spécialisé évoluant rapidement, de nouveaux termes apparaissent dans les documents avant qu'ils n'aient eu le temps d'être consacrés par l'usage ou de faire l'objet d'une norme. Ils dérogent bien souvent des termes connus et acceptés ou sont le fruit d'une utilisation idiosyncratique. Citons, à titre d'exemple, la vedette rapports de gestion - rédaction relevée dans le Répertoire des vedettes-matière (Université Laval, Bibliothèque, 1983). Cette vedette est l'équivalent interlinguistique de Business Report Writing (Library of Congress, 1986) et s'oppose à rapports officiels - rédaction (Government Report Writing). Par ailleurs, rapport de gestion (d'une société) est la traduction que donne le dictionnaire bilingue 
Harrap ${ }^{7}$ de Annual Report (of a company). Ainsi, des documentalistes qui utiliseraient le descripteur rapport de gestion pour chercher des documents, obtiendraient une liste de documents qui porteraient à la fois sur les rapports rédigés dans les sociétés (privées) et sur les rapports annuels selon que ces documents auraient été indexés d'après le premier ou le deuxième sens attribué à rapport de gestion. Ce qui entraîne, évidemment, beaucoup de «bruit» et de confusion lors d'une recherche documentaire.

Comme les documentalistes n'ont ni le temps ni la formation nécessaire pour effectuer les recherches terminologiques nécessaires qui s'imposent dans un tel cas, il paraît, par conséquent, indispensable de confier cette tâche à des terminologues, puis d'incorporer les résultats de leurs recherches et la définition des termes dans la structure des thésaurus, afin d'orienter adéquatement l'indexation et la recherche documentaire et éviter, ainsi, le repérage inutile de documents.

Par ailleurs, les terminologies ne sont, bien souvent, que des listes alphabétiques de termes non regroupés d'aucune façon et ne comportant pas de systèmes de relations. Il est donc impossible, dans un tel cas, d'effectuer des liens entre les termes qui apparaissent comme un ensemble d'éléments disparates. Les terminologies gagneraient, par conséquent, à se rapprocher de la structure du thésaurus qui, en plus de comporter une liste alphabétique structurée à l'intérieur de laquelle les unités sont interreliées par un système de relations, peut comporter, également, divers types de listes structurées (plans de classement, listes thématiques ou hiérarchiques) qui permettent de regrouper les unités d'un même champ notionnel.

Le contenu et la structure des terminologies et des thésaurus sont donc réconciliables dans la mesure où le descripteur acquiert la spécificité du terme et s'accompagne d'une définition et que les terminologies adoptent la structure des thésaurus: système de relations et plan de classement. Pour ce qui est des méthodes de cueillette et de traitement des données, les terminologies et les thésaurus présentent des affinités telles que de simples ajustements seraient nécessaires pour tenir compte de la spécificité de chacun de ces répertoires. Par conséquent, il est possible d'envisager la création d'un répertoire unifié qui réponde, à la fois, à des besoins terminologiques et documentaires.

Le thésaurus terminologique permettant d'allier les points forts des terminologies (définitions) et des thésaurus (classement et relations), présente des avantages certains pour toutes les parties intéressées: comme les documentalistes ne sont pas nécessairement des spécialistes du domaine dans lequel ils effectuent des recherches documentaires, l'apport des définitions, qui précisent le sens des termes, leur facilite grandement la tâche en leur permettant de bien circonscrire un terme. Pour les terminologues et les traducteurs, les relations permettent d'établir des rapports entre des unités voisines et de mieux cerner, ainsi, l'environnement sémantique d'un terme.

\section{B - DESCRIPTION DU THÉSAURUS TERMINOLOGIQUE}

Sager (1982: 211-214) fut l'un des premiers à justifier l'appellation de «thésaurus terminologique» pour désigner le type de répertoire utilisé en terminologie pour consigner les termes des langues de spécialité. Pour lui, cette appellation est préférable à toute autre («vocabulaire classifié ou systématique» ou «glossaire classifié ou systématique») parce qu'elle rend mieux compte de la nature du document. Celui-ci, en effet, se doit de hiérarchiser les termes à l'intérieur d'un champ conceptuel et de les interrelier au moyen d'un système de relations. Le thésaurus lui apparaît alors comme étant le type de répertoire par excellence pour assumer cette double fonction comme en témoignent les thésaurus de langue (tel le Roget's Thesaurus) et les thésaurus documentaires. Aussi en adopte-t-il la structure (et l'appellation) à laquelle il ajoute la définition comme complément. Au déterminé «thésaurus», il accole le déterminant «terminologique» pour le distinguer des 
thésaurus voisins et montrer qu'il consiste en un répertoire de termes. Il en illustre une entrée au moyen du terme «thésaurus terminologique» lui-même (v. Fig. 1).

Ainsi, Sager préconise, à des fins de repérage terminologique, un type de répertoire qui allie la définition aux relations comme celui que nous avions déjà préconisé à des fins documentaires (Larivière, 1985). Aussi, le thésaurus terminologique que nous allons maintenant décrire, tout en conservant cette même structure, pourra tout aussi bien servir au repérage des documents qu'à la consultation terminologique. Nous avons toutefois retenu l'appellation de «thésaurus terminologique» même si ce répertoire doit aussi servir à des fins documentaires. La raison en est que le déterminé «thésaurus» refère à une structure documentaire et le déterminant «terminologique» à un véritable répertoire de termes; en effet, les unités lexicales ont effectivement les caractéristiques du terme tout en agissant comme descripteurs i.e. comme formules d'indexation. Comme ce répertoire constitue une réalité nouvelle, il serait plus approprié de lui donner une désignation propre. Nous avions, quoique timidement, déjà proposé l'appellation de «terminaire» (Larivière, 1986) qui s'insère bien dans le paradigme «vocabulaire-dictionnaire-glossaire» (qui sont, par ailleurs, d'autres types de répertoire) et qui réfère aux objets décrits, soit des termes, tout en ne référant pas, toutefois, à la structure utilisée, soit celle d'un thésaurus. Toutefois, aux fins de cet exposé, retenons, pour le moment, l'appellation de «thésaurus terminologique».

Au plan de la présentation, le thésaurus se compose:

d'une liste thématique hiérarchisée

d'une liste alphabétique structurée

d'un index des termes français

d'un index anglais-français

\section{LA LISTE THÉMATIQUE HIÉRARCHISÉE}

La liste thématique hiérarchisée (v. Fig. 2) constitue un plan de classement de tous les termes. Elle est monohiérarchique i.e. qu'un terme ne peut apparaître que dans une seule hiérarchie. Le classement s'effectue à partir de trois types de rapports: des rapports hiérarchiques de type inclusion-partie (ex.: RAPPORT ANNUEL > BILAN SOCIAL, ÉTATS FINANCIERS, RAPPORT MORAL); des rapports hiérarchiques de type générique-spécifique (ex.: RAPPORT PÉRIODIQUE > RAPPORT ANNUEL, RAPPORT HEBDOMADAIRE); des rapports catégoriels ou facettes (ex.: FRÉQUENCE > RAPPORT PÉRIODIQUE, RAPPORT D'ÉTAPE, de même que TYPES > FRÉQUENCE, ORIENTATION; RAPPORT > TYPES ET COMPOSANTES).

La liste thématique en plus de structurer les termes à l'intérieur de leur champ conceptuel, facilite l'énoncé de la définition qui est fonction du type de rapport qu'occupe le terme à l'intérieur de la liste. Ainsi, les définitions seront de type catégoriel, de type générique ou de type partitif.

\section{LA LISTE ALPHABÉTIQUE STRUCTURÉE}

La liste alphabétique structurée (v. Fig. 3) regroupe tous les termes, descripteurs et non-descripteurs. Les descripteurs, transcrits en capitales (v. $n^{\circ} 050$ ), conformément à la norme de l'AFNOR (1981b: 6), sont structurés en rubriques: DÉFINITION (DF), TRADUCTION (TR), TERMES ÉQUIVALENTS (EP = employé pour), TERME GÉNÉRIQUE (TG), TERMES SPÉCIFIQUES (TS), TERMES ASSOCIÉS (TA) et VOIR AUSSI (VA); les non-descripteurs, transcrits en bas de casse (v. $n^{\circ} 051$ ), sont renvoyés aux descripteurs correspondants à l'aide de l'abréviation EM (employer). 
Les définitions (DF)

Les définitions explicitent la notion. Elles sont constituées du terme supérieur de la hiérarchie ou hyperonyme (genre, tout ou catégorie) et des caractéristiques spécifiques au défini. Dans l'exemple 050 de la Fig. 3, RAPPORT PÉRIODIQUE est le genre prochain. Comme la définition appartient à la structure des terminologies et non à celle des thésaurus, elle ne saurait être traduite, car même dans les terminologies qui s'affichent bilingues, seuls les termes sont traduits et non les définitions.

\section{Les traductions (TR)}

Comme il ne s'agit pas d'un thésaurus bilingue dans lequel les éléments de chacune des composantes comportent des équivalences interlinguistiques (Van Slype, 1987: 56 et AFNOR, 1980), seuls les termes descripteurs sont traduits en anglais afin de faciliter le repérage automatisé des documents du domaine qui abondent en anglais et qui sont compilés dans des banques de données rédigées en anglais. D'ailleurs, il n'est pas nécessaire de traduire les non-descripteurs ou équivalences intralinguistiques (ex. «rapport d'exercice de société anonyme» sous la rubrique EP au numéro 050) puisqu'ils apparaissent sous l'entrée du descripteur qui, lui, est traduit et qu'ils renvoient à la même notion que ce dernier.

Les relations d'équivalence ou préférentielles (EP)

Les termes équivalents ou synonymiques sont ceux qui ont été rejetés comme descripteurs et qui en constituent soit des variantes sémantiques, soit des variantes orthographiques (ex. rapport synthèse et rapport-synthèse), soit des variantes géographiques (ex.: «procès-verbal de gendarmerie» a été écarté au profit de RAPPORT DE POLICE). Le sigle EP indique que le descripteur choisi est «employé pour» les termes rejetés ou nondescripteurs. Ces termes apparaissent toutefois dans la liste alphabétique (v. exemple 051 à la Fig. 3) avec la mention EM (employer) qui renvoie au descripteur retenu.

Le choix des descripteurs s'est effectué en fonction de la fréquence d'apparition d'un terme dans la documentation de base et des usages nord-américains.

Les relations hiérarchiques (v. Fig. 3)

Termes génériques (TG). Les termes génériques ont été puisés à même la liste thématique. $\mathrm{Ce}$ sont les termes hyperonymes ou superordonnés.

Termes spécifiques (TS). Les termes spécifiques constituent des subdivisions hiérarchiques ou catégorielles des descripteurs. Ce sont des hyponymes ou termes subordonnés.

Les relations associatives (v. Fig. 3)

Le thésaurus comporte deux types de relations associatives représentées par les termes associés (TA) et les renvois «voir aussi» (VA). Les termes associés appartiennent à la même hiérarchie que le descripteur à titre de co-hyponymes ou termes coordonnés d'un même hyperonyme (dans l'exemple 050, les termes RAPPORT HEBDOMADAIRE, RAPPORT JOURNALIER, RAPPORT MENSUEL, RAPPORT SEMESTRIEL et RAPPORT TRIMESTRIEL sont des co-hyponymes de RAPPORT ANNUEL et sont avec ce dernier des hyponymes du terme générique ou hyperonyme RAPPORT PÉRIODIQUE.

Les renvois «voir aussi» (VA) sont des termes qui n'entretiennent pas de rapports hiérarchiques avec le descripteur, mais qui sont reliés à ce dernier de façon analogique. Ainsi, RAPPORT ANNUEL est un terme subordonné de RAPPORT PẺRIODIQUE et appartient à sa hiérarchie. Toutefois, par nature, il est aussi, par exemple, une sorte de rapport d'activité; mais il ne peut pas apparaître dans la hiérarchie de RAPPORT 
D'ACTIVITÉ puisque, à ce moment-là, il appartiendrait à plus d'une hiérarchie. Comme nous avons écarté, au départ, la polyhiérarchie, il a donc fallu mettre l'accent sur l'un des traits dominants des descripteurs pour les constituer en hiérarchie (dans le cas de RAPPORT ANNUEL, sur sa périodicité) et faire ressortir leurs autres traits par d'autres types de relations, en l'occurence par les relations associatives de type «voir aussi». Ainsi dans l'entrée de RAPPORT ANNUEL (050), les renvois «voir aussi» indiquent que le rapport annuel peut aussi être considéré comme une sorte de rapport d'activité, de rapport de synthèse, etc. et qu'il serait, par conséquent, utile de consulter ces termes d'indexation pour trouver des ouvrages qui traiteraient du rapport annuel.

\section{INDEX DES TERMES FRANÇAIS (V. FIG. 4)}

Cet index, même s'il semble faire double emploi avec la liste alphabétique structurée, demeure utile à deux égards. D'abord, il permet, en regroupant tous les termes français en quelques pages, d'avoir une vue d'ensemble de ces termes que dilue la liste alphabétique structurée. Ensuite, il permet 'd'effectuer des renvois numériques tant à la liste thématique hiérarchisée (nombres décimaux qui suivent les termes) qu'à la liste alphabétique structurée (nombres entiers qui précèdent les termes). Comme cet index regroupe tous les termes, descripteurs et non-descripteurs, nous avons respecté la typographie habituelle qui sert à les distinguer: descripteurs en capitales et non-descripteurs en bas de casse (AFNOR, 1981b : 16 et Van Slype, 1987:81).

\section{INDEX ANGLAIS-FRANÇAIS (V. FIG. 5)}

Cet index permet de repérer les termes anglais qui n'ont pas, évidemment, d'entrée distincte à l'intérieur de la liste alphabétique structurée puique le thésaurus n'en est pas un bilingue, au sens traditionnel du terme, comportant une liste alphabétique structurée pour chacune des langues envisagées. Cet index ne peut avoir la même présentation que l'index français-anglais puisqu'il n'est pas de même nature que ce dernier; il n'est, en quelque sorte, qu'un lexique anglais-français où les termes anglais, équivalences interlinguistiques des descripteurs français, renvoient à ces derniers qui, eux, apparaissent comme entrées dans la liste alphabétique structurée; comme ils ne sont pas des descripteurs, ils sont typographiés en bas de casse alors que le terme français correspondant, qui est un descripteur, est typographié en capitales.

\section{CONCLUSION}

Ce thésaurus terminologique des documents professionnels se veut être une amorce de conciliation des méthodes utilisées dans la confection des terminologies et des thésaurus qui tendent de plus en plus à se rapprocher8.

Il ne saurait, d'ailleurs, en être autrement. Les coûts de confection des thésaurus et des terminologies sont trop élevés pour pouvoir se payer le luxe d'avoir deux répertoires différents pour un même domaine alors qu'un seul peut répondre aux besoins des utilisateurs quels qu'ils soient.

Il appartient donc aux deux parties intéressées, terminologues et documentalistes, de travailler de concert à produire un répertoire unifié. Pour ce faire, il est nécessaire, il va sans dire, que chaque partie veuille bien abandonner sa chasse gardée pour considérer l'apport de l'autre. Ce qui ne va pas nécessairement se faire sans heurts.

Quoi qu'il en soit, il n'appartient peut-être même pas aux spécialistes de la terminologie et de la documentation de décider si des rapprochements plus intenses doivent s'effectuer entre leur discipline. Il se peut que cette décision appartienne plutôt aux utilisateurs qui, par le biais de l'informatisation, réclameront des outils plus souples et formulés dans un langage clair, plus près des réalités qu'ils côtoient et qu'ils pourront consulter eux-mêmes sans l'aide d'intermédiaires. 


\section{NOTES}

1. On s'est intéressé, entre autres : a) au rôle que pouvait jouer la documentation dans le processus de la terminologie et de la traduction : son importance, ses tâches, ses méthodes (Auger, 1979; Bélanger, 1976, 1980a, 1982 ; Dubuc, 1977, 1980 ; Felber, 1979 ; Kerpan, 1980; Rondeau, 1980); b) au rôle des documentalistes à l'intérieur des services linguistiques: partage des tâches et des responsabilités entre documentalistes et terminologues, bienfaits d'une collaboration entre les deux catégories de spécialistes (Bélanger, 1976, 1980a, $1980 \mathrm{~b}, 1982$ ) ; c) aux sources documentaires : leur nécessité, leur nature, leur organisation, leur valeur, leur degré de fiabilité, la façon d'y accéder et de les exploiter, le bien-fondé des critères pour les évaluer (Bélanger, 1980a, 1982; Rondeau, 1980; Tessier, 1979); d) aux centres de documentation : leur nécessité, la nature et la variétê des services offerts, leur vocation, leur gestion (Auger, 1979; Bélanger, 1980a; Felber, 1979).

2. Une position aussi tranchée ne tient pas compte de la nature des langages documentaires, qui se répartissent en langages classificatoires et en langages combinatoires (Maniez, 1987: 76 et 197), ni des différents niveaux de profondeur d'analyse documentaire auxquels les documents sont soumis: niveau des classes (objets de la classification), des domaines (objets de la catégorisation ou de la pré-sélection), des sujets (objets d'une indexation superficielle) et des CONCEPTS (objets d'une indexation approfondie) (BertrandGastaldy, 1985 : 45 et passim). Donc, la documentation ne s'intéresse pas qu'aux classes. Elle s'intéresse aussi, à un certain niveau d'analyse et tout comme la terminologie, aux concepts représentés par des descripteurs dans les répertoires documentaires que l'on appelle thésaurus.

3. Archimbaud (1979); Fortin et al. (1979); Lazure (1982) et Tessier (1981).

4. C'est la position de Cornog et Landau (1976), Dovbenko et Umanski (1980), Gor'kova (1981), Leska (1981), Maniez (1977) et Sager (1982). Certains ont souligné l'apport de la documentation en terminologie et la nécessité d'une coopération étroite entre les deux disciplines : Dovbenko (1981), Felber (1987:90) et Wüster (1981), ou, inversement, l'apport de la terminologie en documentation: Nikitina (1979) et, une exception au Canada, Dumas et Goyette (1983), deux spécialistes en information documentaire qui se sont appuyés sur les principes et les méthodes de la terminologie pour établir un thếsaurus bilingue en tourisme. D'autres, comme Dahlberg $\{1978,1981 \mathrm{a}, 1981 \mathrm{~b}, 1981 \mathrm{c}, 1983)$, ont établi des principes de classification et de définition qui satisfont aux deux types de répertoires, terminologies et thésaurus.

5. C'est pour cette raison que l'on parle des thésaurus comme étant des langages documentaires créés artificiellement dans lesquels les signes, les descripteurs, ne réfèrent à une réalité que par l'intermédiaire de médias que sont les documents, tandis que, dans les terminologies, les termes renvoient directement au référent.

6. Ainsi dans le Thésaurus international technique (AFNOR, 1981a : 128-129), l'unité «rapport technique» n'a pas été retenue comme descripteur. Pour représenter cette réalité, on a préféré choisir, comme descripteur, l'unité plus vaste de DOCUMENT TECHNIQUE qui est employée pour «rapport technique» et que l'on doit utiliser pour rechercher des documents qui traitent de rapports techniques, faisant ainsi de «rapport technique» un équivalent synonymique au sens documentaire du terme.

7. MANSION, J. E. (1980) : Harrap's New Standard French and English Dictionary, Vol. 4, revised and edited by D. M. Ledésert \& R. P. Ledésert, London (England) : Harrap, 1980. s.y. report.

8. Certains auteurs (Dovbenko, 1980:11) ne peuvent, par ailleurs, concevoir la création d'un répertoire qu'en intégrant les caractéristiques des terminologies et des thésaurus à l'intérieur d'un système automatisé de gestion de l'information, ce qui semble être la tendance actuelle en URSS. Aux États-Unis, certains thésaurus comportent, en plus des composantes habituelles, une composante glossaire, quoique non intégrée aux autres composantes : c'est le cas du thésaurus médical Medlars (cité par Maniez, 1977: IV-45) et du Criminal Justice Glossary (Cornog, 1976).

\section{OUVRAGES CONSULTÉS}

AFNOR (1980): Documentation, Principes directeurs pour l'établissement des thésaurus multilingues, Norme expérimentale Z 47-101, Paris, AFNOR, avril 1980.

AFNOR (1981a): Thésaurus international technique, Paris, AFNOR, 1981.

AFNOR (1981b) : Documentation : Règles d'établissement des thésaurus monolingues, Norme française homologuée NF Z 47-100, Paris, AFNOR, décembre 1981.

ARCHIMBAUD, Robert (1979): «Classement des données terminologiques et documentaires», Secrétariat d'État du Canada, pp. 283-303.

AUGER, Pierre (1979) : «Rapports entre terminologie et documentation», Office de la langue française, pp. 661-665.

AUGER, Pierre, ROUSSEAU, Louis-Jean et al. (1978) : Méthodologie de la recherche terminologique, Québec, Office de la langue française, (Études, recherches et documentation).

BÉLANGER, Nycole (1976): «Fonction terminologie et fonction documentation», Meta 21-1 (mars 1976), pp. 68-71. 
BÉLANGER, Nycole (1980a) : «Avant-propos», La documentation. Meta 25-1, (mars 1980), pp. 5-6.

BÉLANGER, Nycole (1980b) : «Le documentaliste de services linguistiques», La documentation, Meta 25-1 (mars 1980), pp. 21-27.

BÉLANGER, Nycole (1982) : «La documentation spécialisée en terminologie et l'apport du spécialiste dans ce domaine», Actes du troisième colloque OLF-STQ de terminologie : le rôle du spécialiste dans les travaux de terminologie, Sainte-Marguerite (Québec), du 13 au 15 février 1980, Québec, Éditeur officiel du Québec, pp. 45-58.

BERTRAND-GASTALDY, Suzanne (1985): Guide d'apprentissage $n^{\circ} 3$, Les différents types de traitement analytique et de langages documentaires, Notes de cours BLT 6103: Traitement et analyse documentaire, Université de Montréal, École de bibliothéconomie et des sciences de l'information, hiver 1985, $71 \mathrm{p}$.

BOUTIN-QUESNEL, Rachel et al. (1985) : Vocabulaire systématique de la terminologie, Québec, Les publications du Québec, 38 p. (Cahiers de l'Office de la langue française).

CLAS, André et Jean BAUDOT (1986): «B A T E M, Une banque de terminologie sur micro-ordinateur», INFOTERM, Actes du Deuxième Symposium d'Infoterm, Travail dans le cadre d'un réseau de terminologie; Coopération internationale dans le travail terminologique, Vienne, 1985-04-14/17, München, K. G. Saur, (Infoterm Series; 8), pp. 376-389.

CORNOG, Martha et Herbert B. LANDAU (1976) : «Application of lexicographic techniques to the production of a national criminal justice glossary and thesaurus», Information Politics, Proceedings of the ASIS 39th Annual Meeting, vol. 13, San Francisco, Calif., Oct. 4-9, 1976. pp. 773-782.

DAHLBERG, Ingetraut (1978): Ontical structures and universal classification, Bangalore, Sạroda Ranganathan Endowment, $64 \mathrm{p}$.

DAHLBERG, Ingetraut (1981a) : «Conceptual definitions for INTERCONCEPT», International Classification 8-1, pp. 16-22.

DAHLBERG, Ingetraut (1981b) : «On the structure of definitions». INFOTERM, pp. 568-570.

DAHLBERG, Ingetraut (1981c) : «Les objets, les notions, les définitions et les termes», RONDEAU et FELBER, réd, (1981): pp. 221-282.

DAHLBERG, Ingetraut (1983): «Terminological definitions : characteristics and demands», Problèmes de la définition et de la synonymie en terminologie, Actes du colloque international de terminologie, Québec 2327 mai 1982, Québec, GIRSTERM, pp. 16-34.

DOVBENKO, M. A. (1981) : «The role of terminology in science and technology», INFOTERM, pp. 131-137.

DOVBENKO, M. A. et A. N. UMANSKI (1980) : «A comparative analysis of information retrieval thesauri and normalized scientific and technical terminologies with the aim of their coordinated development». International Forum on Information and Documentation 5-4, pp. 9-11.

DUBUC, Robert (1977): «Terminologie et traduction», $8^{\circledR}$ Congrès de la Fédération internationale des traducteurs, Montréal, 14-18 mai 1977.

DUBUC, Robert (1980): «Pour une saine gestion de la documentation en terminologie et en traduction», la documentation, Meta 25-1 (mars 1980), pp. 11-20.

DUMAS, Daniel et Marie GOYETTE (1983): Apports de la terminologie dans le processus de traduction d'un thésaurus en tourisme, Université de Montréal, École de bibliothéconomie et des sciences de l'information, avril 1983.

FELBER, Helmut (1979) : «Gestion d'un centre de documentation terminologique», Office de la langue française, pp. 325-362.

FELBER, Helmut (1987): Manuel de terminologie, Paris, UNESCO.

FORTIN, Jean-Marie et al. (1979): «Est-il souhaitable de définir des normes canadiennes pour les systèmes de classement des données documentaires ?», Secrétariat d'État, pp. 283-303.

GOR'KOVA, V. I. (1981) : «Terminology development, the application of methods used in Informatics», INFOTERM, pp. 503-513.

INFOTERM (1981): «Problèmes théoriques et méthodologiques de la terminologie», Actes du colloque international, Moscou, 27-30 novembre 1979. Munich, K. G. Saur.

KERPAN, Nada. «La documentation et les services linguistiques», La documentation, Meta 25-1 (mars 1980), pp. 7-10.

LARIVIERE, Louise (1985): Thésaurus RÉDAC, rédaction professionnelle, projet, Université de Montréal, École de bibliothéconomie et des sciences de l'information, décembre 1985, $112 \mathrm{f}$.

LARIVIERE, Louise (1986): Terminaire des documents professionnels : I - Le rapport, Université de Montréal, École de bibliothéconomie et des sciences de l'information, avril-mai 1986, $73 \mathrm{f}$.

LAZURE, N. (1982): «Le thésaurus TERMI», Terminologies for the Eighties, with a special section : 10 years on Infoterm, Munich, K. G. Saur, 1982, (Infoterm series; 7), pp. 367-372.

LESKA, B. M. (1981): «The connections between systems of concepts and thesauri», INFOTERM, pp. 583590.

LIBRARY OF CONGRESS (1986) : Library of Congress Subject Headings, $10^{\mathrm{e}}$ éd., Washington, DC, Library of Congress, $2 \mathrm{v}$. 
MANIEZ, Jacques (1977): «Terminologies et thésaurus: divergences et convergences», Terminologies 76 : Colloque international, Paris - La Défense, $15-18$ juin 1976, Paris, La maison du dictionnaire, pp. IV-39 - IV-50.

MANIEZ, Jacques (1987): Les langages documentaires et classificatoires : conception, construction et utilisation dans les systèmes documentaires, Paris, Les éditions d'organisation, (Systeme d'information et de documentation).

NIKITINA, S. E. (1979): «Thésaurus d'information comme moyen de systématiser la terminologie» [compte rendu résumé d'un colloque sur la terminologie tenu à Léningrad en mars 1974], Travaux de terminologie 1 , Québec, GIRSTERM, Université Laval, pp. 61-67.

OFFICE DE LA LANGUE FRANÇ.AISE (1979): Actes du 6e Colloque international de terminologie, Pointeau-Pic (Québec), 2 au 6 octobre 1977, Québec, Éditeur officiel du Québec.

RONDEAU, Guy (1980): «Terminologie et documentation», La documentation, Meta 25-1, (mars 1980), pp. $152-170$.

RONDEAU, Guy et Helmut FELBER, réd. (1981) : Textes choisis de terminologie : I - Fondements théoriques de la terminologie, Québec, GIRSTERM

SAGER, J. C. (1982) : «Terminological thesaurus : a more appropriate designation or a deprecated synonym ?», Social Sciences Information Studies 2, pp. 211-214.

SECRÉTARIAT D'ÉTAT DU CANADA (1979): Actes du Colloque canadien sur les fondements d'une méthodologie générale de la recherche et de la normalisation en terminologie et en documentation, Ottawa, 16-18 février 1976, Québec, GIRSTERM, Université Laval.

SPARCK JONES, Karen et Martin KAY (1973) : Linguistics and Information Science, New York, Academic Press.

TESSIER, Philippe (1979) : «Est-il souhaitable de définir des normes canadiennes quant à la valeur des sources documentaires; si oui, quelles sont les étapes à franchir?», SECRÉTARIAT D'ÉTAT DU CANADA, Pp. 305-312.

TESSIER, Philippe (1981) : «Le thésaurus TERMI», INFOTERM, pp. 284-287.

UNIVERSITÉ LAVAL, BIBLIOTHEQUE (1983) : Répertoire de vedettes-matière, Vol. 2 et 3, 9e éd., Québec, Bibliothèque de 1'Université Laval.

VAN SLYPE, Georges (1987) : Les langages d'indexation : conception, construction et utilisation dans les systèmes documentaires, Paris, Les éditions d'organisation, (Systèmes d'information et de documentation).

WÜSTER, E. (1981) : «L'étude scientifique générale de la terminologie, zone frontalière entre la linguistique, la logique, l'ontologie, l'informatique et les sciences des choses», RONDEAU et FELBER, pp. 55-114. 